\title{
Clinical Features and CT Imaging Analysis of Hepatic Sinuscase-Syndrome and Budd-Chiari Syndrome
}

\author{
Feng Dai ${ }^{1, *}$, Wei Qiao ${ }^{2, *}$, Zheng Kang $\mathbb{D}^{2}$, Yan $\mathrm{Chen}^{3}, \mathrm{Kang} \mathrm{Li}^{2}$, Wenrong Shen $\mathbb{D}^{2}$, Xiuming Zhang ${ }^{2}$ \\ 'Department of Interventional Radiology, The Second Hospital of Nanjing, Nanjing University of Chinese Medicine, Nanjing, People's Republic of \\ China; ${ }^{2}$ Department of Radiology, Jiangsu Cancer Hospital \& Jiangsu Institute of Cancer Research \& The Affiliated Cancer Hospital of Nanjing Medical \\ University, Nanjing, People's Republic of China; ${ }^{3}$ Department of Pathology, Jiangsu Cancer Hospital \& Jiangsu Institute of Cancer Research \& The \\ Affiliated Cancer Hospital of Nanjing Medical University, Nanjing, People's Republic of China
}

*These authors contributed equally to this work

Correspondence: Xiuming Zhang, Email zhangxiuming360@163.com; Wenrong Shen, Email jszlyyct@sohu.com

\begin{abstract}
Objective: This study aimed to analyze the clinical features and computed tomography (CT) manifestations of hepatic sinusoidal obstruction syndrome (HSOS) induced by Gynura segetum, a Chinese herbal medicine, so as to improve the clinical understanding and diagnosis of the disease.

Methods: Relevant clinical and laboratory parameters and CT imaging data of 20 patients with HSOS confirmed by liver biopsy were retrospectively analyzed and compared with 16 patients with Budd-Chiari syndrome (BCS).

Results: Levels of alanine aminotransferase, aspartate aminotransferase, alkaline phosphatase, and glutamyl transpeptidase increased significantly $(\mathrm{p}<0.05)$ in HSOS patients compared to the BCS patients, while the albumin level and prothrombin time, which are indicators of liver synthesis function, decreased and prolonged significantly, respectively. All 20 patients with HSOS had manifestations of ascites and heterogeneous hypoattenuation on CT, including 18 cases (90\%) with heterogeneous enhancement (characteristic map-like enhancement), 17 (85\%) with hepatomegaly, 18 (90\%) with gallbladder wall oedema, and 16 (80\%) with stenosis of main hepatic veins and characteristic "clover-like" enhancement at the second porta hepatis.

Conclusion: Both HSOS and BCS are post-sinusoidal portal hypertension, but have different etiologies and durations. Although they both cause liver congestion, the clinical manifestation of HSOS is acute liver injury. The CT manifestations are characterized by ascites, map-like enhancement and heterogeneous hypoattenuation of the liver parenchyma, and stenosis of the main hepatic veins. BCS is often found in the stage of decompensated liver cirrhosis, resulting in liver shrinkage, splenomegaly, and ascites.
\end{abstract}

Keywords: hepatic sinusoidal obstruction syndrome, Gynura segetum, Budd-Chiari syndrome, computed tomography

\section{Background}

Hepatic sinusoidal obstruction syndrome (HSOS), also known as hepatic veno-occlusive disease, is a vascular disease in which endothelial cells in the hepatic sinusoids, small hepatic veins, and interlobular veins undergo oedema and necrosis due to multiple reasons, leading to microthrombosis, subsequent congestive liver injury, and portal hypertension. ${ }^{1}$ HSOS mostly occurs after hematopoietic stem cell transplantation. In China, it mainly occurs after the administration of pyrrolidine alkaloid (PA)-containing herbs, such as Gynura segetum. ${ }^{2}$ HSOS is clinically manifested by symptoms such as liver pain, ascites, and jaundice, and is often diagnosed as hepatitis, liver cirrhosis, Budd-Chiari syndrome (BCS), or unexplained liver disease.

Portal hypertension can occur due to pre-sinusoidal, sinusoidal, and post-sinusoidal obstruction and lead to liver injury. Except for heart-induced congestive hepatopathy $(\mathrm{CH})$, post-sinusoidal obstruction is mainly divided into two categories according to the level of obstruction: (1) HSOS: at the level of sinusoids and terminal venules, (2) BCS: from the hepatic vein to the superior end of inferior vena cava.,

Due to the lack of specificity in the clinical manifestation of HSOS, early diagnosis is difficult and it is often confused with BCS in clinical practice. Only a few studies analyzed their clinical imaging features. This study retrospectively 
analyzed 20 cases of HSOS and explored the clinical testing parameters and computed tomography (CT) imaging features of HSOS to improve the clinical understanding and diagnosis of this disease.

\section{Objective}

This study aimed to analyze the clinical features and CT manifestations of HSOS caused by Gynura segetum, a Chinese herbal medicine, so as to improve the clinical understanding and diagnosis of this disease.

\section{Materials and Methods}

\section{Clinical Data}

This study retrospectively analyzed 36 patients with post-sinusoidal portal hypertension. Among them, 20 were patients with HSOS, and 16 were patients with BCS. These patients were referred to or sought Medical care in the Department of Hepatology, Nanjing Second Hospital from January 2016 to December 2019 due to abdominal distension, anorexia, asthenia, and hepatic dysfunction.

All HSOS patients had a specific history of administration Gynura segetum, including 13 cases of oral administration and 7 cases of drinking Gynura segetum wine. The earliest symptoms can appear as early as 3 days, and the longest can take up to one year after taking Gynura segetum. Approximately 50\% of patients developed symptoms between 15 and 30 days after taking Gynura segetum. Their diagnoses were confirmed by liver biopsy.

The diagnostic criteria of HSOS were as follows: (1) hepatomegaly and hepatalgia; (2) jaundice; (3) ascites or weight gain over $2 \%$ of baseline. This study also included patients with at least two of the three symptoms described above but without other types of liver disease, such as viral, autoimmune, or metabolic liver disease. 15 of the included HSOS cases underwent ultrasound-guided liver biopsy, and 5 underwent transjugular vein liver biopsy.

The experimental data collection was approved by the Ethics Committee of Jiangsu Cancer Hospital and the Second Hospital of Nanjing. This study was not considered to impose an additional burden on patients, cause injury to patients or affect their treatment, and did not violate patients' privacy. Before the start of the study, each patient was informed that relevant images and clinical data during the treatment were used for Research and analysis. Patients' personal information was not disclosed and the consent of the patients was obtained. We confirm that the study complies with the Declaration of Helsinki.

\section{Image Analysis}

All 20 HSOS patients and 16 BCS patients underwent plain, enhanced, and delayed CT scans. 5 BCS patients also underwent digital substruction angiography (DSA). All imaging data were retrospectively analyzed by two experienced radiologists. According to published literature, ${ }^{5} \mathrm{CT}$ images of the HSOS cases were mainly analyzed for the following parameters and features: liver volume (head-tail $>18 \mathrm{~cm}$ ), hepatic vein stenosis (right hepatic vein diameter $<4.5 \mathrm{~mm}$ ), gallbladder wall thickening (wall thickness $>3 \mathrm{~mm}$ ), degree of ascites, and map-like heterogeneous enhancement of liver parenchyma in the venous and delayed phases. The degree of enhancement was divided into three levels according to the range of heterogeneous enhancement: Level 1 is the mild heterogeneous enhancement in a small liver segment; Level 2 is the confluent heterogeneous enhancement in most liver segments; Level 3 is the diffuse confluent heterogeneous enhancement throughout the entire liver. ${ }^{6}$ BCS was caused by a hepatic venous obstruction in 5 of the patients, inferior vena cava obstruction in 3 of the patients, and 8 cases suffered from a combination of the two conditions.

\section{Statistical Analysis}

Data analysis was performed using statistical software SPSS 20.0 (IBM Corp., Armonk, NY, USA), and all variables were tested for normality using the Shapiro-Wilk test. For variables that have a normal distribution, an independent samples $t$-test was performed. For variables that do not have a normal distribution, a nonparametric rank-sum test was performed. A $p<0.05$ indicates a statistically significant difference. 


\section{Results}

\section{Results of Clinical and Laboratory Tests}

There were 20 patients with HSOS, 16 males and 4 females, aged 39-74 years with an average age of $61.0 \pm 7.42$ years. There were 16 patients with BCS, 9 males and 7 females, aged 22-64 years, with an average age of $45.88 \pm 11.88$ years (Table 1).

The red blood cell, hemoglobin, and white blood cell counts in HSOS patients were all within the normal range, but were significantly higher than those in patients with BCS $(p<0.05)$. There was no significant difference in the platelet counts between the two groups (Table 1). The levels of indicators of liver damage in HSOS patients, aspartate aminotransferase and alanine aminotransferase, were higher than the normal levels and were significantly higher than those in BCS patients $(p<0.05)$. The levels of alkaline phosphatase and glutamyl transpeptidase, indicators of cholestasis, were also increased in HSOS patients, and the difference was statistically significant between the two groups $(p<0.05)$. At the same time, the albumin level and prothrombin time, which reflect liver synthesis function, were decreased and prolonged, respectively, in the patients with HSOS, and the difference between the two groups was statistically significant $(p<0.05)$ (Table 1).

\section{Results of Imaging Examination and Pathological Examination}

Ascites, heterogeneous hypoattenuation, and heterogeneous enhancement are the most important imaging manifestations of HSOS (Figure 1A-C). The prevalence was as follows: ascites $(100 \%, 20 / 20)$, heterogeneous hypoattenuation $(100 \%$, $20 / 20)$, and heterogeneous enhancement $(95 \%, 18 / 20)$ (Table 2). Furthermore, heterogeneous enhancement was divided into three levels according to the enhancement range as shown in Figure 2. The prevalence of the three manifestations was significantly lower in BCS patients than in HSOS patients.

At the same time, the imaging manifestations of hepatomegaly, gallbladder wall thickening, and hepatic main veins stenosis were significantly more obvious in HSOS patients than in BCS patients. Their prevalence rates of these three manifestations in HSOS patients were 85\% (17/20), 90\% (18/20), and 80\% (16/20), respectively, which were significantly different from those of the BCS group $(p<0.05)$ (Table 2). Despite the narrowing of the main hepatic veins, the liver parenchyma surrounding the three hepatic veins exhibited enhancement in the venous and delayed phases, which

Table I Laboratory Parameters of Hepatic Sinuscirrhotic Syndrome and Budd-Chiari Syndrome

\begin{tabular}{|c|c|c|c|c|}
\hline Parameter & HSOS $(n=20)$ & $\operatorname{BCS}(n=16)$ & Statistic & $\boldsymbol{P}$ \\
\hline Age* (Mean \pm SD) & $61 \pm 7.42$ & $45.88 \pm 11.88$ & - & $<0.05$ \\
\hline $\operatorname{Sex}^{\mathrm{a}}$ & & & l & 0.16 \\
\hline Male & $16(80 \%)$ & $9(56.3 \%)$ & & \\
\hline Female & $4(20 \%)$ & $7(43.8 \%)$ & & \\
\hline Red blood cell ${ }^{* *}($ Mean $\pm S D), 10^{12} / \mathrm{L}$ & $5.04 \pm 0.73$ & $4.30 \pm 0.43$ & 3.559 & $<0.05$ \\
\hline Haemoglobin** (Mean \pm SD), g/L & $150.55 \pm 19.73$ & $126.38 \pm 20.1 \mid$ & 3.622 & $<0.05$ \\
\hline White blood cell** (Mean \pm SD), $10^{9} / \mathrm{L}$ & $7.89 \pm 3.01$ & $4.19 \pm 1.51$ & 4.474 & $<0.05$ \\
\hline Platelet* (Mean \pm SD), $10^{9} / \mathrm{L}$ & $109.25 \pm 51.21$ & $103.44 \pm 66.56$ & -0.557 & 0.577 \\
\hline Alanine aminotransferase* (Mean $\pm \mathrm{SD}), \mathrm{U} / \mathrm{L}$ & $103.99 \pm|| 2.4 \mid$ & $18.36 \pm 6.68$ & - & $<0.05$ \\
\hline Aspartate aminotransferase* (Mean $\pm \mathrm{SD}), \mathrm{U} / \mathrm{L}$ & $123.90 \pm 151.38$ & $24.06 \pm 7.54$ & - & $<0.05$ \\
\hline Total bilirubin* (Mean \pm SD), $\mu \mathrm{mol} / \mathrm{L}$ & $85.69 \pm 98.92$ & $23.21 \pm 15.37$ & - & $<0.05$ \\
\hline Albumin** (Mean \pm SD), g/L & $32.82 \pm 4.07$ & $40.11 \pm 6.12$ & -4.282 & $<0.05$ \\
\hline Alkaline phosphatase* (Mean \pm SD), U/L & $|76.76 \pm 77.3|$ & $79.86 \pm 23.97$ & - & $<0.05$ \\
\hline Glutamyl transpeptidase* (Mean $\pm S D$ ), U/L & $|86.02 \pm| 39.81$ & $52.77 \pm 19.17$ & - & $<0.05$ \\
\hline Creatinine ${ }^{* *}$ (Mean $\left.\pm S D\right), \mu \mathrm{mol} / \mathrm{L}$ & $95.20 \pm 33.42$ & $64.63 \pm 15.67$ & 3.367 & $<0.05$ \\
\hline Urea nitrogen* (Median $\pm \mathrm{IQR}$ ), $\mathrm{mmol} / \mathrm{L}$ & $6.33,4.27$ & $5.00,2.05$ & - & $<0.05$ \\
\hline Prothrombin time* (Mean \pm SD), S & $16.46 \pm 3.78$ & $13.53 \pm 1.79$ & - & $<0.05$ \\
\hline International normalized ratio* (Median $\pm \mathrm{IQR}$ ) & $1.37,0.26$ & $1.22,0.16$ & - & $<0.05$ \\
\hline
\end{tabular}

Notes: **Normal distribution ( $t$-test) with statistical significance $(P<0.05)$; ${ }^{*}$ Non-normal distribution (rank sum test) with statistical significance $(P<0.05)$; ${ }^{\text {Fisher }}$ exact test. 


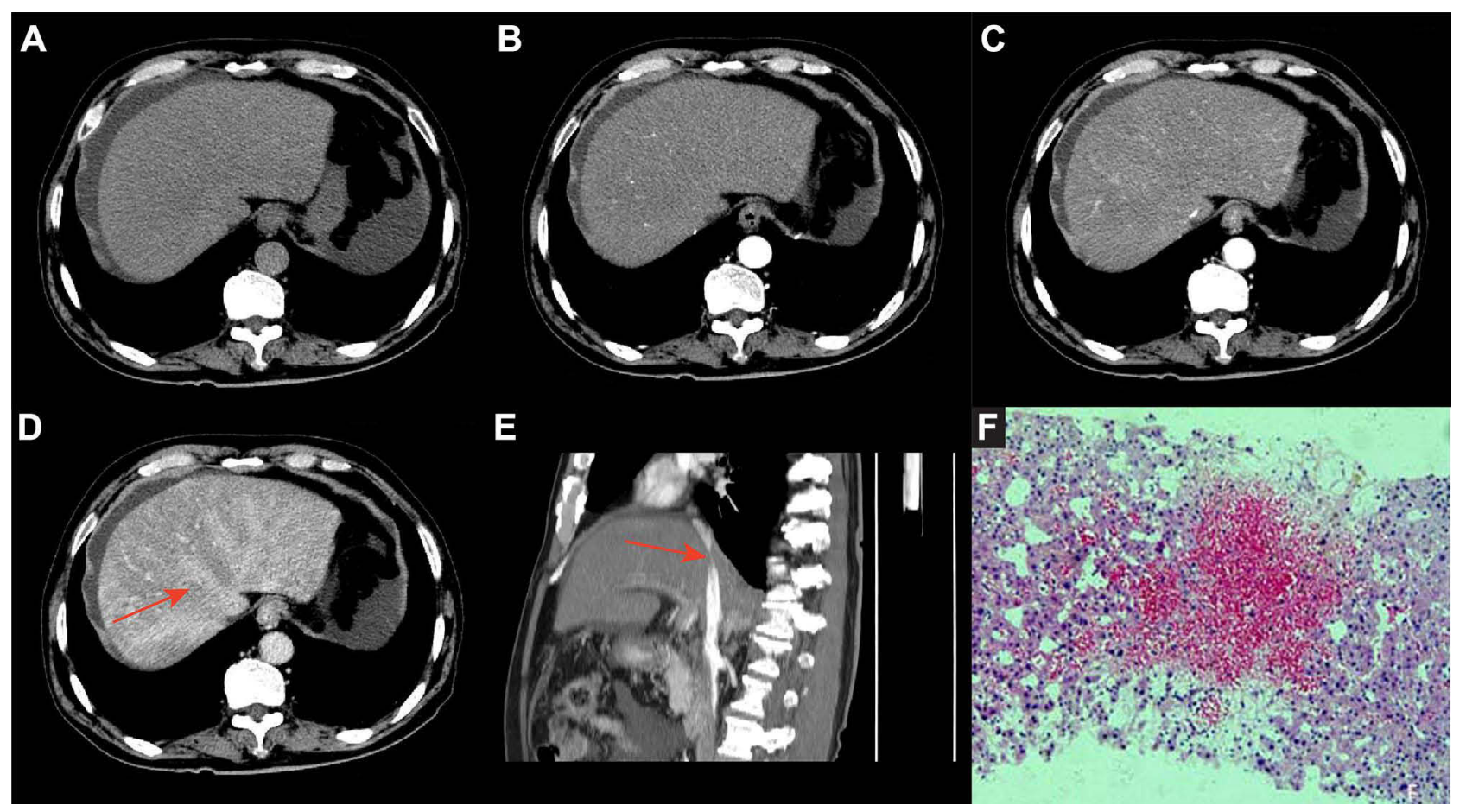

Figure I CT manifestations and pathological pictures of hepatic sinusoidal obstruction syndrome. (A-C) Imaging manifestations of hepatic sinusoidal obstruction syndrome on plain CT scans, arterial phase CT scans, and venous phase CT scans. (D) Clover-like enhancement in the second porta hepatis (arrowhead) on delayed-phase CT scans. (E) Sagittal multiplanar reconstruction showing stenosis of the hepatic inferior vena cava (arrowhead). (F) HE staining (I0xI0) showed congestion around the central vein, with thinning and necrosis of adjacent hepatocytes.

was highlighted in the background of decreased enhancement of liver parenchyma elsewhere. This type of enhancement was referred to as clover-like enhancement (Figure 1D and E), a characteristic imaging manifestation of HSOS. The Hematoxylin and Eosin (HE) staining of the liver biopsy showed congestion around the central vein with thinning and necrosis of adjacent hepatocytes (Figure 1F).

\section{Discussion}

\section{Etiological and Pathological Basis of HSOS}

HSOS often occurs after a bone marrow transplant or after taking certain Chinese herbal medicines containing PA, especially in patients with a history of taking Gynura segetum. Chemotherapies used in colorectal liver metastases have also been identified as a risk factor for HSOS, particularly the usage of oxaliplatin (OX). ${ }^{7}$ Studies have shown that endothelial cell damage in the hepatic sinusoids and central vein region, intimal hyperplasia, and fibrosis of venules cause the obstruction of blood outflow and subsequent acute hepatocyte necrosis. Related pathological studies also support the

Table 2 Imaging Features of Hepatic Sinuscirrhotic Syndrome and Budd-Chiari Syndrome

\begin{tabular}{|l|l|l|}
\hline & HSOS n/N (\%) & BCS n/N (\%) \\
\hline Hepatomegaly & $16 / 20(80.0)$ & $6 / 16(37.5)$ \\
Gallbladder wall thickening & $18 / 20(90.0)$ & $7 / 16(43.75)$ \\
Splenomegaly & $5 / 20(25.0)$ & $14 / 16(87.5)$ \\
Ascites & $20 / 20(100)$ & $7 / 16(43.75)$ \\
Heterogeneous enhancement & $18 / 20(90.0)$ & $5 / 16(31.2)$ \\
Heterogeneous hypoattenuation & $20 / 20(100)$ & $4 / 16(25.0)$ \\
Narrowing of main hepatic veins & $15 / 20(75.0)$ & $5 / 16(31.2)$ \\
\hline
\end{tabular}




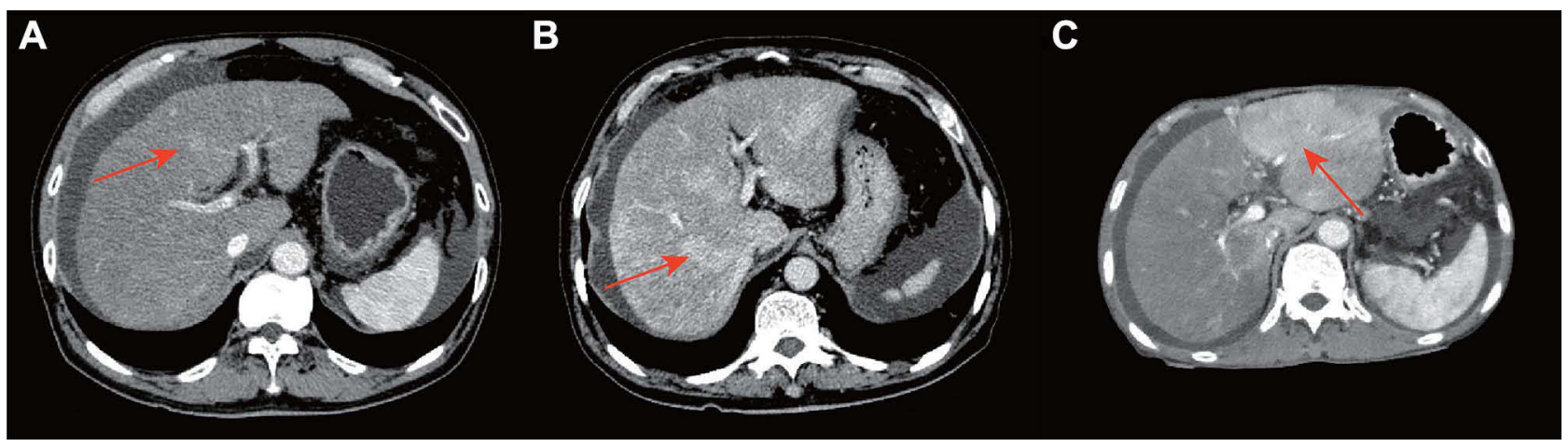

Figure 2 Different levels of heterogeneous enhancement on CT images of patients with hepatic sinusoidal obstruction syndrome. (A) Level I, mild and limited patchy enhancement (arrowhead). (B) Level 2, moderate confluent patchy enhancement (arrowhead). (C) Level 3, severe diffuse confluent patchy enhancement (arrowhead).

aforementioned view, namely, necrosis of hepatocytes in the center of the liver lobules, and the dilation and bleeding of sinusoids around the central vein. ${ }^{8}$ Although liver biopsy is the golden standard for the diagnosis of HSOS, familiarity with typical imaging features by clinicians and imaging physicians is still helpful for the early diagnosis and treatment of the disease.

\section{Clinical and Laboratory Parameters of HSOS}

HSOS and BCS are the two most common diseases characterized by post-sinusoidal portal hypertension due to the obstruction of hepatic venous outflow. However, they involve different sites, which are keys to analyzing their clinical and imaging features. ${ }^{9}$

The most common clinical manifestations of HSOS are abdominal distension, loss of appetite, fatigue, and jaundice, which are mainly caused by liver dysfunction as a result of acute liver cell injury, central venous congestion, haemorrhage, and hepatocellular necrosis. ${ }^{10}$ In this study, it was observed that the levels of aspartate and alanine aminotransferase were significantly higher in the HSOP patients than those in the BCS patients, suggesting that acute liver damage was more severe in the patients with HSOS, while BCS is a chronic pathological process with the well-compensated liver. The levels of alkaline phosphatase and glutamyl transpeptidase were also significantly higher in the patients with HSOS than those in the patients with BCS, due to the obstruction of the bile duct and bile secretion impairment caused by acute liver injury. Studies have suggested that patients with HSOS may have renal dysfunction and their prognosis of kidney injury is poor, which was consistent with an observation that the levels of creatinine and urea nitrogen were significantly higher in patients with HSOS than those in patients with BCS in this study.

Coagulation dysfunction plays an important role in the occurrence and development of HSOS. Animal studies have shown that Gynura segetum can reduce the number of platelets and inhibit platelet aggregation in rats, leading to prolonged coagulation time. ${ }^{11}$ This study showed that the prothrombin time and the international normalized ratio of the patients with HSOS were elevated, which were significantly different from BCS patients. It is possible that the white blood cell, red blood cell, and platelet counts of routine blood tests are lower in BCS patients than those in HSOS patients due to the chronic course of BCS causes splenomegaly and hypersplenism. However, only white blood cell and red blood cell counts were observed significantly lower in BCS patients than HSOS patients, while there was no significant difference in platelet counts between the two groups, which may be attributed to the lesser effect of Gynura segetum on the blood system of diseased individuals compared with that of healthy individuals. Given the high incidence of pleural effusion and ascites in patients with HSOS, secondary infection cannot be ruled out as a possible cause of the high white blood cell counts in these patients. 


\section{HSOS Imaging Features}

Ascites and hepatic parenchyma heterogeneous hypoattenuation are the most common CT findings in HSOS and were present in all HSOS patients. The heterogeneous enhancement of the liver parenchyma is an important imaging feature of HSOS, and its pathological basis was the obstruction of blood outflow due to the necrosis of hepatocytes in the center of the liver lobules with together the dilation and bleeding of sinusoids around the central veins. Therefore, in contrast to the background of diffuse hypoattenuation of liver parenchyma, the scattered or confluent areas of heterogeneous patchy and the map-like enhancement around the small branches of the portal vein on the portal venous phase CT scans expand over time. ${ }^{12}$ Different degrees and scopes of enhancements were clearly observed in this study (Figure 2). Zhou et al ${ }^{13}$ showed that the degree of patchy and the map-like enhancement were significantly correlated with the clinical severity of HSOS and were sensitive diagnostic indicators of HSOS.

Hepatomegaly is also a common imaging manifestation of HSOS. Although nonspecific, it is suggested that acute hepatocyte injury is caused by the obstruction of acute hepatic venous outflow, which is different from chronic outflow tract obstruction or stenosis in BCS. The acute obstruction of hepatic venous outflow is associated with liver congestion and the thinning and necrosis of hepatocytes, and may also be caused by hypoproteinemia. At the same time, the thickening of the gallbladder wall is also attributed to the increased resistance in venous blood flow, which is different from the thickening and oedema caused by cholecystitis. The stenosis of main hepatic veins has been widely accepted as an important diagnostic imaging feature of HSOS. ${ }^{14}$ This study observed $75 \%$ of the HSOS patients with three major hepatic veins stenosis. Erturk et $\mathrm{al}^{15}$ proposed that a right hepatic vein diameter $<4.5 \mathrm{~mm}$ highly indicates HSOS and attributed it to the severe obstruction of hepatic sinusoidal and venous outflow. In addition, the stenosis of the hepatic inferior vena cava, which is the cause of liver parenchymal swelling and the obstruction of hepatic venous outflow, was also a unique imaging manifestation of HSOS (Figure 1E).

The CT scans of some cases showed that the enhancement of hepatic tissues surrounding the three main hepatic veins was greater than that of other hepatic tissues, but the enhancement was concentrated in the second porta hepatis and exhibited a clover-like pattern (Figure 1D). Therefore, this enhancement was referred to as a clover-like enhancement in this study. In addition, the liver parenchyma adjacent to the three main hepatic veins contains many small draining veins, which are still in a state of perfusion function and have relatively small hemodynamic changes, thereby leading to a relatively normal enhancement of the nearby liver parenchyma. ${ }^{13}$

\section{Differential Diagnosis of HSOS}

HSOS and BCS are the two most common diseases characterized by post-sinusoidal portal hypertension. Although they share similar liver clinical manifestations and histological features, there are significant differences between them in the following aspects: (1) In terms of etiology, most patients with HSOS have a clear history of administration of pyrrole alkaloid-containing drugs (such as Gynura segetum), or undergo radiotherapy and chemotherapy, and also have an acute liver injury, while most patients with BCS have a history of unexplained chronic liver cirrhosis; (2) In terms of clinical manifestations, HSOS has a short course of disease, and the clinically manifests are acute hepatocyte injury, bile duct obstruction, and bile secretion impairment, while BCS has a relatively longer course of disease and its clinical manifests are mostly chronic compensated cirrhosis or chronic decompensated cirrhosis with hypersplenism; (3) In terms of imaging features, HSOS is mainly characterized by ascites, hepatomegaly, typical map-like heterogeneous enhancement, and clover-like enhancement accompanied by the stenosis of the main hepatic veins or hepatic inferior vena cava. However, the blood flow of HSOS patients is still unobstructed. In contrast, BCS patients mainly have CT manifestations of chronic liver disease, such as liver cirrhosis and splenomegaly, marked occlusion or stenosis of the hepatic veins and/or the inferior vena cava with an open collateral circulation, such as the retroperitoneal azygos or hemiazygos veins (Figure 3).

This study had the following limitations: (1) All the HSOS patients were diagnosed by biopsy, but the BCS patients were diagnosed only by imaging and clinical features without biopsy; (2) The imaging analysis was only based on clinical routine plain CT scans and contrast-enhanced CT scans, while did not involve magnetic resonance image analysis, which left room for further improvement of the comprehensive evaluation of HSOS; (3) Both oxaliplatin and Gynura segetum can cause HSOS, but the HSOS caused by oxaliplatin mostly occurs $6-8$ cycles after administration, 


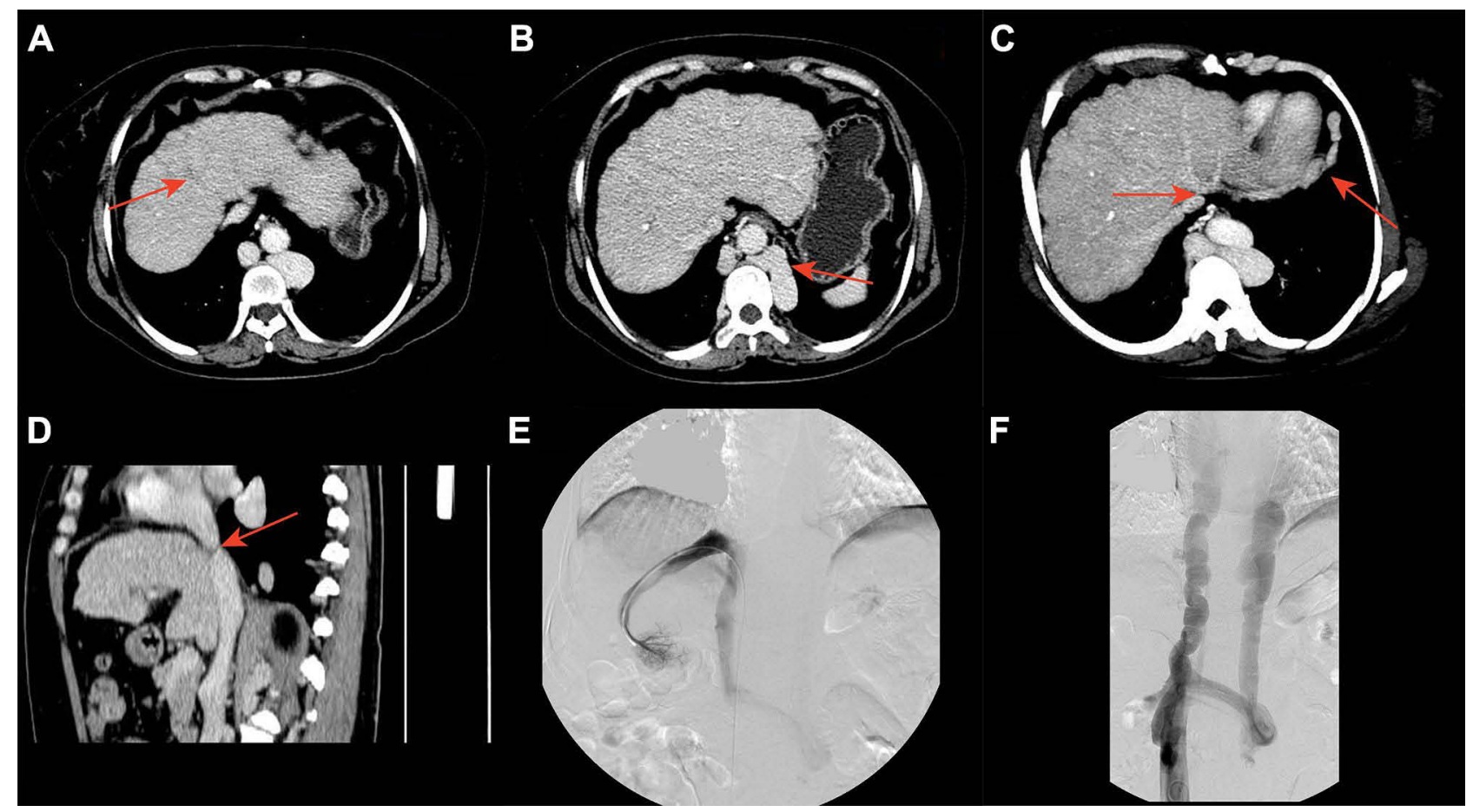

Figure 3 CT manifestations of Budd-Chiari syndrome. (A) Uneven strengthening of liver parenchyma (arrowhead) on delayed-phase CT scans. (B) Retroperitoneal azygos and hemiazygos vein tortuous and expansion on delayed-phase CT scans (arrowhead). (C) The left and middle hepatic veins do not flow into the inferior vena cava. Cardiophrenic angle varice (arrowhead). (D) Membranous occlusion of inferior vena cava (arrowhead). (E) The right hepatic vein flows directly into the inferior vena cava on Digital subtraction angiography. (F) Retroperitoneal azygos and hemiazygos vein tortuous and expansion on Digital subtraction angiography.

which has obvious dose-dependent characteristics. It has been confirmed by the relevant literature ${ }^{7}$ that the HSOS caused by Gynura segetum was not dose-dependent. In addition, in this study, the sample size was small, so the two samples cannot be distinguished from each other on the performance of contrast-enhanced CT.

This study summarized and analyzed the clinical and imaging features of HSOS, and provided deeper insights into the diagnosis and treatment of HSOS for clinical and imaging physicians. The main findings were as follows: (1) All HSOS patients had a history of administration of Gynura segetum or similar drugs, and relevant clinical and laboratory parameters suggested the acute hepatocyte injury and the obstruction of bile conduct; (2) The typical CT imaging features of HSOS were ascites, heterogeneous hypoattenuation, map-like heterogeneous enhancement, stenosis of the main hepatic veins and the hepatic inferior vena cava, and clover-like enhancement of the second porta hepatis. These clinical and laboratory characteristics and CT image features provide a sufficient basis for the early clinical diagnosis of HSOS.

\section{Author Contributions}

All the authors made significant contributions to the work presented here, whether in conception, study design, execution, data acquisition, data analysis and interpretation, or in all these aspects. All authors took part in drafting, revising or critically reviewing the article; gave final approval of the version to be published; have agreed on the journal to which the article has been submitted; and agree to be accountable for all aspects of the work.

\section{Funding}

This work was supported by the Roentgen special fund for image research of Jiangsu Medical Association (SYH32011500008 2021003) and the Jiangsu Cancer Hospital Young Talents Plan (Jiangsu China).

\section{Disclosure}

Feng Dai and Wei Qiao are co-first authors for this study. The authors declare no conflicts of interest in this work. 


\section{References}

1. Helmy A. Review article: updates in the pathogenesis and therapy of hepatic sinusoidal obstruction syndrome. Aliment Pharmacol Ther. 2006;23 (1):11-25. doi:10.1111/j.1365-2036.2006.02742.x

2. Lin G, Wang JY, Li N, et al. Hepatic sinusoidal obstruction syndrome associated with consumption of Gynura segetum. J Hepatol. 2011;54 (4):666-673. doi:10.1016/j.jhep.2010.07.031

3. Soni N, Israrahmed A, Singh P, et al. Caput medusa: a sign of portal hypertension on case of chronic Budd-Chiari syndrome. BMJ Case Rep. 2021;14(4):e242076. doi:10.1136/bcr-2021-242076

4. Balducci G, Sterpetti AV, Ventura M. A short history of portal hypertension and of its management. J Gastroenterol Hepatol. 2016;31(3):541-545. doi:10.1111/jgh.13200

5. Shi TT, He J, Zhuge YZ, et al. CT feature of hepatic veno-occlusive disease caused by Gynura segetum. J Pract Radiol. 2016;32:875-878.

6. Kan XF, Ye J, Rong XX, et al. Diagnostic performance of contrast-enhanced CT in pyrrolizidine alkaloids-induced hepatic sinusoidal obstructive syndrome. Sci Rep. 2016;6(1):37998. doi:10.1038/srep37998

7. Cayet S, Pasco J, Dujardin F, et al. Diagnostic performance of contrast-enhanced CT-scan in sinusoidal obstruction syndrome induced by chemotherapy of colorectal liver metastases: radio-pathological correlation. Eur J Radiol. 2017;94:180-190. doi:10.1016/j.ejrad.2017.06.025

8. Yang XQ, Ye J, Li X, et al. Pyrrolizidine alkaloids-induced hepatic sinusoidal obstruction syndrome: pathogenesis, clinical manifestations, diagnosis, treatment, and outcomes. World J Gastroenterol. 2019;25(28):3753-3763. doi:10.3748/wjg.v25.i28.3753

9. Ulas DB, Soley S, Yusuf B. Hepatic venous outflow obstruction: three similar syndromes. World J Gastrenterol. 2007;13(13):1912-1927. doi:10.3748/wjg.v13.i13.1912

10. Wang JY, Gao H. Tusanqi and hepatic sinusoidal obstruction syndrome. J Dig Dis. 2014;15(3):105-107. doi:10.1111/1751-2980.12112

11. Deleve LD, Shulman HM, McDonald GB. Toxic injury to hepatic sinusoids: sinusoidal obstruction syndrome. Semin Liver Dis. 2002;22(1):27-42. doi: $10.1055 / \mathrm{s}-2002-23204$

12. Kan XF, Ye J, Rong XX, et al. Diagnostic performance of contrast-enhanced CT in pyrrolizidine Alkaloids-induced hepatic sinusoidal obstructive syndrome. Sci Rep. 2016;6(11):1-8.

13. Zhou H, Wang YX, Lou HY, et al. Hepatic sinusoidal obstruction syndrome caused by herbal medicine: CT and MRI features. Korean J Radiol. 2014;15(2):218-225. doi:10.3348/kjr.2014.15.2.218

14. Sun ZY, Kang JM, Zhang YC. Hepatic veno-occlusive disease related to Gynura segetum: a case report. Medicine (Baltimore). $2018 ; 97(17): \mathrm{e} 0552$. doi:10.1097/MD.0000000000010552

15. Erturk SM, Mortele KJ, Binkert CA, et al. CT features of hepatic veno occlusive disease and hepatic graft-versus-host disease in patients after hematopoietic stem cell transplantation. AJR. 2006;186(6):1497-1501. doi:10.2214/AJR.05.0539

International Journal of General Medicine

\section{Dovepress}

\section{Publish your work in this journal}

The International Journal of General Medicine is an international, peer-reviewed open-access journal that focuses on general and internal medicine, pathogenesis, epidemiology, diagnosis, monitoring and treatment protocols. The journal is characterized by the rapid reporting of reviews, original research and clinical studies across all disease areas. The manuscript management system is completely online and includes a very quick and fair peer-review system, which is all easy to use. Visit http://www.dovepress.com/testimonials.php to read real quotes from published authors.

Submit your manuscript here: https://www.dovepress.com/international-journal-of-general-medicine-journal 